\title{
Association between periodontitis and serum c-reactive protein levels
}

\author{
Rafael-Paschoal Esteves-Lima ${ }^{1}$, Christian-Santiago Reis ${ }^{2}$, Francisco Santirocchi-Júnior ${ }^{2}$, Lucas-Guimarães \\ Abreu $^{3}$, Fernando-Oliveira Costa ${ }^{4}$
}

\author{
${ }^{1} \mathrm{PhD}$ in Periodontics. Faculty of Dentistry of Federal University of Minas Gerais, Av, Antônio Carlos, 6627, Belo Horizonte (MG), \\ 31270-901, Brazil \\ ${ }^{2}$ Graduate in Dentistry. Faculty of Dentistry of Federal University of Minas Gerais, Av, Antônio Carlos, 6627, Belo Horizonte \\ (MG), 31270-901, Brazil \\ ${ }^{3} \mathrm{PhD}$ in Pediatric Dentistry. Faculty of Dentistry of Federal University of Minas Gerais, Av, Antônio Carlos, 6627, Belo Horizonte \\ (MG), 31270-901, Brazil \\ ${ }^{4} \mathrm{PhD}$ in Epidemiology. Faculty of Dentistry of Federal University of Minas Gerais, Av, Antônio Carlos, 6627, Belo Horizonte \\ (MG), 31270-901, Brazil
}

Correspondence:

Faculty of Dentistry of Federal University of Minas Gerais

Av, Antônio Carlos, 6627

Belo Horizonte (MG), 31270-901, Brazil

rafaelpaschoalesteves@yahoo.com.br

Esteves-Lima RP, Reis CS, Santirocchi-Júnior F, Abreu LG, Costa FO. Association between periodontitis and serum c-reactive protein levels. J Clin Exp Dent. 2020;12(9):e838-43.

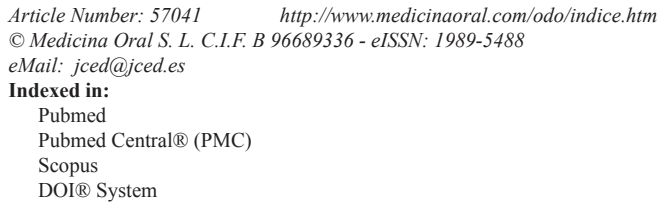




\section{Introduction}

Periodontitis is a progressive inflammatory disease of bacterial etiology characterized by gum bleeding, increased probing depth, and attachment loss. The aggression caused by bacterial biofilm stimulates an immune and destructive response to periodontal tissues, leading to collagen destruction, apical migration of junctional epithelium and loss of alveolar bone (1).

$\mathrm{C}$-reactive protein (CRP) is an acute phase protein considered a non-specific and highly sensitive inflammatory marker, produced by liver cells in response to various forms of injury to the body (2). Systemic infection, trauma and hypoxia are associated with an increased production of CRP. Moreover, inflammatory cytokines, such as interleukin 6 , interleukin 1 and tumor necrosis factor $\alpha$ can trigger the production of CRP by hepatocytes (3). Factors, such as smoking, obesity, diabetes and pregnancy have been associated with high serum levels of CRP (2).

The translocation of bacteria and bacterial products of oral cavity can induce a systemic inflammatory process, characterized by high levels of pro-inflammatory cytokines, including increased levels of CRP (4). CRP has also been considered a significant risk factor for many systemic diseases, such as cardiovascular disease and type 2 diabetes $(3,5)$. In this scenario, the hypothesis that individuals with periodontitis present modified levels of CRP has been raised.

Although, the fact that periodontitis may be associated with changes in the levels of inflammatory markers makes the systemic impact of periodontal alterations relevant, few studies have assessed the relationship between periodontitis and altered levels of CRP. Scientific evidence on this relationship is still controversial and reduction in CRP levels is not always observed after periodontal therapy (6). Additionally, almost all studies have evaluated the relationship between periodontitis and CRP in individuals with some comorbidity, especially cardiovascular disease. Therefore, the objective of this study was to evaluate the association between periodontitis and serum levels of CRP.

\section{Material and Methods}

-Sampling, setting and period of data collection

The sample of this cross-sectional study was composed of 100 individuals attending the outpatient clinic of Periodontology of the Dental School of the Federal University of Minas Gerais, Belo Horizonte, Brazil. Individuals were randomly selected from September 2017 to December 2018.

Individuals who were 18 years or older and those presenting a minimum of 12 teeth were included. Individuals living with the human immunodeficiency virus, pregnant women, individuals who had undergone periodontal treatment within the last three months, individuals who had undergone anti-inflammatory or antimicrobial therapy within the last three months, those with diabetes, individuals with any contraindication to clinical periodontal examination and individuals reporting any systemic health condition were excluded.

The reporting of this manuscript conforms to The Strengthening the Reporting of Observational Studies in Epidemiology (STROBE) guidelines (7). Approval of the Ethics and Research Committee was obtained (CAAE 37241214.2.0000.5097). This study was conducted in accordance with the Helsinki Declaration. Participants were informed about the objectives and the methods of the study. Those who accepted to participate signed an informed consent form.

-Sociodemographic and behavioral characteristics

Participants' data on sociodemographic and behavioral characteristics were collected by means of an interview during which a structured questionnaire was used. Data on sex, age, family income (number of wages of all members of the family who were economically active), schooling (number of years of studying) and smoking (smokers, non-smokers) (8) were collected.

-Periodontal clinical examination

Clinical examination was carried out in four sites (mesial, buccal, distal and lingual) of all teeth for analysis of the following periodontal parameters for periodontal diagnosis: probing depth (PD), bleeding on probing (BOP) and clinical attachment level (CAL). During clinical examination, a manual periodontal probe (UNC15 , Hu-Friedy () ) was used.

Three examiners (F.S.J., C.S.R. and R.P.E.L.), previously trained and calibrated, conducted the examination of the participants. Values of inter and intra-examiner agreement assessed by the interclass correlation coefficient were above 0.86 for PD and CAL.

Teeth with changes in gingival morphology, teeth with extensive caries lesions, those with poor restorative procedures and teeth, for which the determination of the limit between the enamel and cementum was unfeasible, were excluded (9).

-Definition of periodontitis and assessment of the prevalence

The criteria for periodontitis definition was the presence of $\geq 4$ teeth having $\geq 1$ sites with $\mathrm{PD} \geq 4 \mathrm{~mm}$ and $\mathrm{CAL} \geq 3$ $\mathrm{mm}$ associated with BOP (2).

-Medical data

A blood test of each participant was ordered for quantification of CRP. The collection of blood samples and the analysis of the samples of all individuals were carried out providing a period of eight hours of fasting. CRP values less than $3 \mathrm{mg} / \mathrm{L}$ were considered normal. Values greater than or equal to $3 \mathrm{mg} / \mathrm{L}$ were considered altered (10). Thus, participants were divided into two groups, according to the serum levels of CRP: individuals with normal CRP and individuals with altered CRP. 
Weight and height were measured for body mass index (BMI) calculation. Participants could be assigned to the following subgroups according to their BMI: underweight (BMI below 18.5), normal weight (BMI between 18.5 and 24.9), overweight (BMI between 25 and 29.9) and obese (BMI above 29.9).

-Statistical analyses

Data analyzis was performed using the Statistical Package for the Social Sciences (SPSS Inc., version 23.0, Armonk, USA). Descriptive analyzis was conducted. The Mann-Whitney test was used to compare individuals with normal CRP and those with altered CRP with respect to sociodemographic variables (sex, age, family income and schooling) as well as smoking and BMI. The Mann-Whitney test was also used to compare individuals with normal CRP and those with altered CRP with respect to periodontitis (percentage of individuals with or without periodontitis). Finally, a regression analysis evaluating the association between periodontitis and CRP alterations controlling for confounding variables (age, smoking and BMI) was carried out. In all analyzis, results with a probability lower than $5 \%$ were considered significant $(P<0.05)$.

\section{Results}

Comparisons between the groups with normal and altered CRP levels regarding the sociodemographic variables (sex, age, family income and schooling) as well as smoking, BMI and periodontitis are presented in Table 1. No significant difference between groups regarding sex, age, family income, schooling and smoking was observed. Individuals with altered CRP presented a mean BMI of 33.0, while individuals with normal CRP presented a mean BMI of 26.0. A significant difference between groups was observed $(P<0.001)$. The prevalence of periodontitis among individuals with altered CRP $(66.7 \%)$ was significantly higher than among individuals with normal CRP $(37.9 \%)(P=0.008)$.

The regression analyzis demonstrated that the levels of CRP among individuals with periodontitis were 1.72 times higher than the levels of CRP among individuals without periodontitis (confidence interval=1.02 - 2.93;

Table 1: Comparisons between individuals with normal and altered CRP with respect to independent variables.

\begin{tabular}{|c|c|c|c|c|}
\hline Variable & Total sample & Normal CRPa) & Altered CRPa) & $P$-value \\
\hline $\mathrm{N}$ & 100 & 58 & 42 & \\
\hline Age (yr.) & $39.4( \pm 11.2)$ & $39.1( \pm 11.6)$ & $39.9( \pm 10.8)$ & 0.999 \\
\hline $\operatorname{Sex}(\%)$ & & & & 0.163 \\
\hline Male & $24(24 \%)$ & $17(29.3 \%)$ & $07(16.7 \%)$ & \\
\hline Female & $76(76 \%)$ & $41(70.7 \%)$ & $35(83.3 \%)$ & \\
\hline Family income & & & & 0.435 \\
\hline$\leq 5$ salary & $89(89 \%)$ & $51(87.9 \%)$ & $38(90.5 \%)$ & \\
\hline$>5$ salary & $11(11 \%)$ & $07(12.1 \%)$ & $04(9.5 \%)$ & \\
\hline Schooling (yr.) & & & & 0.091 \\
\hline$\leq 8$ & $38(38 \%)$ & $17(29.3 \%)$ & $21(50.0 \%)$ & \\
\hline $9-12$ & $44(44 \%)$ & $28(48.3 \%)$ & $16(38.1 \%)$ & \\
\hline$>12$ & $18(18 \%)$ & $13(22.4 \%)$ & $05(11.9 \%)$ & \\
\hline Smoking & $10(10 \%)$ & $05(8.6 \%)$ & $05(11.9 \%)$ & 0.738 \\
\hline $\operatorname{BMI}\left(\mathrm{Kg} / \mathrm{m}^{2}\right)$ & $28.8( \pm 5.7)$ & $26.0( \pm 4.3)$ & $33.0( \pm 6.2)$ & $<0.001^{\text {a) }}$ \\
\hline CRP (mg/L) & $4.7( \pm 6.8)$ & $1.4( \pm 0.8)$ & $9.5( \pm 8.7)$ & $<0.001^{\text {a) }}$ \\
\hline Periodontitis & $50(50 \%)$ & $22(37.9 \%)$ & $28(66.7 \%)$ & $0.008^{\text {a) }}$ \\
\hline
\end{tabular}

yr.: year, CRP: c-reactive protein, BMI: body mass index.

a) Statistically significant difference 
$P=0.042$ ), regardless of the influence of the cofounding variables age, smoking and BMI (Table 2). The analyzis also showed that the levels of CRP among obese individuals were 3.48 times higher than the levels of CRP among underweight individuals/individuals with normal weight (confidence interval $=1.56-7.75 ; P=0.002$ ). white blood cell count and platelet-lymphocyte ratio mediated $8 \%, 13 \%$ and $16 \%$ of the association between severe periodontitis and diabetes. Other studies have described findings similar to our results on the association between periodontitis and serum levels of CRP (12-16). One study (17), for instance, demonstrated that the pre-

Table 2: Regression analysis evaluating the association of periodontitis and CRP alteration, controlling for confounding variables (age, smoking and body mass index.

\begin{tabular}{|c|c|c|c|c|}
\hline & $\begin{array}{c}\text { Ratio (95\% CI) } \\
\text { Non-adjusted }\end{array}$ & $P$-value & $\begin{array}{c}\text { Ratio (95\% CI) } \\
\text { Adjusted }\end{array}$ & $P$-value \\
\hline \multicolumn{5}{|l|}{ Periodontitis } \\
\hline No & 1 & $0.007^{\text {a) }}$ & 1 & $0.042^{\text {a) }}$ \\
\hline Yes & $2.00(1.20-3.32)$ & & $1.72(1.02-2.93)$ & \\
\hline \multicolumn{5}{|l|}{ Age (yr.) } \\
\hline$\leq 35$ & 1 & & 1 & \\
\hline$>35$ to $<42$ & $0.94(0.51-1.70)$ & 0.838 & $1.01(0.59-1.71)$ & 0.971 \\
\hline$>43$ & $0.85(0.49-1.46)$ & 0.562 & $0.60(0.36-0.98$ & 0.055 \\
\hline \multicolumn{5}{|l|}{ Smoking } \\
\hline No & 1 & 0.565 & 1 & 0.484 \\
\hline Yes & $1.21(0.62-2.37)$ & & $1.24(0.67-2.29)$ & \\
\hline \multicolumn{5}{|l|}{ BMI } \\
\hline Underweight/normal weight & 1 & & 1 & \\
\hline Overweight & $1.36(0,90-2.09)$ & 0.146 & $1.16(0.73-1.84)$ & 0.524 \\
\hline Obese & $4.00(1.83-8.69)$ & $<0.001$ a) & $3.48(1.56-7.75)$ & 0.002 a) \\
\hline
\end{tabular}

yr.: year, CI: confidence interval, BMI: body mass index.

a) Statistically significant difference

\section{Discussion}

CRP, a marker for acute inflammation, is produced by the liver as a result of various types of injuries, including infectious illnesses. Inflammatory mediators arising from periodontitis may stimulate hepatocytes to produce CRP. Among these mediators, interleukin-1, interleukin-6 and tumor necrosis factor alpha are particularly involved in this process (2). In this sense, periodontal infection may lead to systemic inflammation with a significant increase in CRP levels. The results of the present study showed that individuals with periodontitis presented a significantly higher level of CRP in comparison with individuals without periodontitis. These results allow one to accept the hypothesis that periodontitis is associated with a higher production of this nonspecific inflammatory marker with far-reaching effects on the individual affected by periodontitis. In one previous study (11), the authors evaluated how systemic inflammation mediated the association between periodontitis and glycemic status. The results of their study showed that CRP, sence of Porphyromonas gingivalis increases CRP levels in nearly $20 \%$. It should be emphasized that almost all studies analyzing the association between periodontitis and serum CRP levels have evaluated individuals with any systemic changes, such as cardiovascular disease, diabetes or rheumatoid arthritis. The presence of systemic changes can be a confounding factor in assessing the impact of periodontitis on CRP levels. In our study, only individuals without any systemic alteration participated. The exclusion of individuals who had reported the presence of any systemic alteration during an interview strengthens the present study. However, it is possible that some unknown systemic changes may be present in some individuals in the sample, which is a limitation. Additionally, the severity of periodontitis may have a direct impact on the levels of CRP. One study (18) demonstrated that CRP levels increase with the severity of periodontitis. Cases of very progressive and extensive periodontitis are associated with higher CRP levels $(12,13,15,19-21)$. As regards periodontal therapy, howe- 
ver, intervention studies show discrepancies whether periodontal treatment has an impact on the reduction of serum CRP levels (14,15,21-23).

In addition to the periodontal infection, other factors may be associated with the increased production of CRP. This fact may explain why some individuals without periodontitis presented significant changes in CRP levels. Factors such as age, smoking and obesity have been associated with alterations of the levels of CRP (24). Older individuals, smokers and individuals with obesity may have increased levels of this marker. This is the reason why regression analyzis assessing the relationship between periodontitis and CRP levels, controlling for these confounders has been conducted herein.

In this study, individuals with normal CRP and altered CRP were roughly equal in age, and the mean age of the sample was not high. CRP concentration increases with age, probably due to the increased incidence of subclinical pathological conditions. Age is a highly relevant factor that should be taken into account when CRP is assessed. This result diverges from the fact that a higher age is associated with increased levels of CRP (24). On the other hand, the prevalence and severity of periodontitis may also increase with age (25). Therefore, age is a confounding factor that should be evaluated in studies in which the association between periodontitis and serum CRP levels is assessed. The similarity between groups of individuals with normal and altered CRP serum levels regarding age is a strong point of the present study, favoring the analysis of the relationship between periodontitis and CRP.

Herein, the regression analyzis demonstrated that obese individuals presented significantly higher levels of CRP in comparison with underweight individuals/normal weight individuals. The scientific literature has shown that obesity is associated with high levels of CRP because the secretion of inflammatory cytokines is increased in individuals affected by obesity (26). Obesity has also been associated with periodontitis. The secretion of cytokines by the adipose tissue leads to a more exacerbated inflammatory response with a strong impact on the pathogenesis of periodontitis (27).

Smoking has been associated with high levels of CRP (24). Smoking is also a risk factor for periodontitis. Tobacco use plays an important role in the pathogenesis of inflammatory periodontal disease. Smokers present greater prevalence and severity of periodontitis (2). Herein, no significant difference between groups regarding the number of smokers was observed. Moreover, the regression analyzis also demonstrated that the levels of CRP among smokers were not significantly different in comparison with the levels of the non-smokers. The low number of individuals who were smokers and participated in this study may explain the similarity between groups regarding the smoking habit. In addition, CRP levels appear to be higher in women and in individuals with low socioeconomic status $(28,29)$. Importantly, in this study, the groups of individuals with normal and altered levels of CPR were similar in relation to sex, family income and schooling.

The definition of the criteria for periodontitis diagnosis is an important methodological step in studies in which the association between periodontal disease and levels of inflammatory markers is evaluated. The criteria used for the diagnosis of periodontitis may have a great impact on the prevalence of the disease (30). The definition of periodontitis should not underestimate or overestimate the disease. The characteristics of the periodontal condition of the sample should also be considered. Individuals with differences in the extension and severity of the periodontal disease may present different systemic behaviors. This fact should be considered when interpreting the results and conclusions of the different studies. Additionally, a thorough periodontal examination is necessary to establish an appropriate diagnosis. In the present study, a complete periodontal clinical examination was performed to evaluate the periodontal parameters and a robust diagnostic criterion was used to assess the systemic impact of periodontitis.

In this study, individuals with periodontitis presented higher levels of CRP in comparison with individuals without periodontitis. This positive association reinforces the theory that periodontitis has a significant influence on the levels of inflammatory biomarkers, suggesting that periodontal infection can lead to a systemic impact, favoring the development and aggravation of other pathologies. However, additional studies, in particular intervention and longitudinal studies, with special attention to confounding factors, are needed to further assess the association between periodontitis and serum levels of CRP.

\section{References}

1. Albandar JM. Global risk factors and risk indicators for periodontal diseases. Periodontol 2000. 2002;29:177-206.

2. Gomes-Filho IS, Coelho JMF, Cruz SS, Passos JS, Freitas COT, Farias NSA, et al. Chronic periodontitis and C-reactive protein levels. J Periodontol. 2011;82:969-978.

3. Bansal T, Dhruvakumar D, Pandey A. Comparative evaluation of C-reactive protein in peripheral blood of patients with healthy gingiva, gingivitis and chronic periodontitis: A clinical and particle-ennanced turbidimetric immuno-analysis. J Indian Soc Periodontol. 2014;18:739-743.

4. Tawfig A. Effects of non-surgical periodontal therapy on serum lipids and C-reactive protein among hyperlipidemic patients with chronic periodontitis. J Int Soc Prevent Communit Dent. 2015;5:49-56.

5. Demmer RT, Trinquart L, Zuk A, Fu BC, Blomkvist J, Michalowicz $\mathrm{BS}$, et al. The influence of anti-infective periodontal treatment on C-reactive protein: a systematic review and meta-analysis of randomized controlled trials. PLoS One. 2013;8:e77441.

6. Quintero AJ, Chaparro A, Quirynen M, Ramirez V, Prieto D, Morales $\mathrm{H}$, et al. Effect of two periodontal treatment modalities in patients with uncontrolled type 2 diabetes mellitus: A randomized clinical trial. J Clin Periodontol. 2018;45:1098-1106. 
7. von Elm E, Altman DG, Egger M, Pocock SJ, Gøtzsche PC, Vandenbroucke JP. STROBE Initiative. The Strengthening the Reporting of Observational Studies in Epidemiology (STROBE) statement: guidelines for reporting observational studies. J Clin Epidemiol. 2008;61:344-9.

8. Tomar SL, Asma S. Smoking-attributable periodontitis in the United States: findings from NHANES III. J Periodontol. 2000;71:743-751.

9. Costa FO, Cota LOM, Costa JE, Pordeus IA. Periodontal disease progression among young subjects with no preventive dental care: A 52-month follow-up study. J Periodontol. 2007;78:198-203.

10. Ndrepepa G, Siegmund B, Salvatore C, Fusaro M, King L, Kastrati $\mathrm{A}$, et al. C-reactive protein and prognosis in women and men with coronary artery disease after percutaneous coronary intervention. Cardiovasc Revasc Med. 2013;14:264-269.

11. Torrungruang K, Ongphiphadhanakul B, Jitpakdeebordin S, Sarujikumjornwatana S. Mediation analysis of systemic inflammation on the association between periodontitis and glycaemic status. J Clin Periodontol. 2018;45:548-556.

12. Bolla V, Kumari PS, Munnangi SR, Kumar DS, Durgabai Y, Koppolu P. Evaluation of Serum C-reactive Protein Levels in Subjects with Aggressive and Chronic Periodontitis in Comparison with Healthy Controls: A Clinico-biochemical Study. Int J App Basic Med Res. 2017;7:121-124.

13. Chandy S, Joseph K, Sankaranarayanan A, Issac A, Babu G, Wilson B, et al. Evaluation of C-Reactive Protein and Fibrinogen in Patients with Chronic and Aggressive Periodontitis: A Clinico-Biochemical Study. J Clin Diagn Res. 2017;11:ZC41-ZC45.

14. de Souza AB, Okawa RT, Silva CO, Araújo MG. Short-term changes on $\mathrm{C}$-reactive protein (CRP) levels after non-surgical periodontal treatment in systemically healthy individuals. Clin Oral Investig. 2017;21:477-484.

15. Ramich T, Asendorf A, Nickles K, Oremek GM, Schubert R, Nibali L, et al. Inflammatory serum markers up to 5 years after comprehensive periodontal therapy of aggressive and chronic periodontitis. Clin Oral Investig. 2018;22:3079-3089.

16. Sezgin Y, Bulut Ş, Bozalığlu S, Sezgin A. Levels of High-Sensitivity C-Reactive Protein in Heart Transplant Patients With and Without Periodontitis. Exp Clinical Transplant. 2019;17:123-127.

17. Winning L, Patterson CC, Cullen KM, Stevenson KA, Lundy FT, Kee F, et al. The association between subgingival periodontal pathogens and systemic inflammation. J Clin Periodontol. 2015;42:799-806. 18. Torrungruang K, Katudat D, Mahanonda R, Sritara P, Udomsak A. Periodontitis is associated with elevated serum levels of cardiac biomarkers-soluble ST2 and C-reactive protein. J Clin Periodontol. 2019;46:809-818.

19. Gupta B, Sawhney A, Patil N, Yadav M, Tripathi S, Sinha S, et al. Effect of Surgical Periodontal Therapy on Serum C-reactive Protein Levels Using ELISA in Both Chronic and Aggressive Periodontitis Patient. J Clin Diagn Res 2015;9:ZC01-5.

20. Podzimek S, Mysak J, Janatova T, Duskova J. C-Reactive Protein in Peripheral Blood of Patients with Chronic and Aggressive Periodontitis, Gingivitis, and Gingival Recessions. Mediators Inflamm. 2015;564858.

21. Mysak J, Podzimek S, Vasakova J, Mazanek J, Vinsu A, Duskova J. C-reactive protein in patients with aggressive periodontitis. J Dent Sci. 2017;12:368-374.

22. Paraskevas S, Huizinga JD, Loos BG. A systematic review and meta-analyses on c-reactive protein in relation to periodontitis. J Clin Periodontol. 2008;35:277-290.

23. Escobar GF, Abdalla DR, Beghini M, Gotti VB, Rodrigues Junior V, Napimoga MH, et al. Levels of Pro and Anti-inflammatory Citokynes and C-Reactive Protein in Patients with Chronic Periodontitis Submitted to Nonsurgical Periodontal Treatment. Asian Pacific J Cancer Prev. 2018;19:1927-1933.

24. Yamazaki K, Honda T, Oda T, Ueki-Maruyama K, Nakajima T, Yoshie $\mathrm{H}$, et al. Effect of periodontal treatment on the C-reactive protein and proinflammatory cytokine levels in Japanese periodontitis patients. J Periodontal Res. 2002;40:53-58.
25. American Academy of Periodontology. Position paper: epidemiology of periodontal diseases. J Periodontol. 1996;67:935-945.

26. Fantuzzi G, Mazzone T. Adipose Tissue and Atherosclerosis: Exploring the Connection. Arterioscler Thromb Vasc Biol. 2007;27:9961003.

27. Pataro AL, Costa FO, Cortelli SC, Cortelli JR, Souza ACD, Abreu MHNG, et al. Influence of Obesity and Bariatric Surgery on the Periodontal Condition. J Periodontol. 2012;83:257-266.

28. Woloshin S, Schwartz LM. Distribution of C-reactive protein values in the United States. N Engl J Med. 2005;352:1611-1613.

29. Kinane DF, Riggio MP, Walker KF, MacKenzie D, Shearer B. Bacteraemia following periodontal procedures. J Clin Periodontol. 2005;32:708-713.

30. Costa FO, Guimarães AN, Cota LOM, Pataro AL, Segundo TK, Cortelli SC, et al. Impact of different periodontitis case definitions on periodontal research. J Oral Sci. 2009;51:199-206.

\section{Ethics}

Approval of the Ethics and Research Committee was obtained (CAAE 37241214.2.0000.5097).

\section{Source of funding}

No funding to this article was reported.

\section{Authors' contributions}

All authors participated in the preparation of the present case-control study, including the methodological development and the search in the scientific literature for relevant studies on the subject. The authors Rafael Paschoal Esteves Lima, Christian Santiago Reis and Francisco Santirocchi Júnior were responsible for the selection of the sample. The authors Christian Santiago Reis and Francisco Santirocchi Júnior were responsible for the data collection. Data analysis was performed by the authors Rafael Paschoal Esteves Lima, Lucas Guimarães Abreu and Fernando Oliveira Costa. The authors Rafael Paschoal Esteves Lima, Christian Santiago Reis and Francisco Santirocchi Júnior were responsible for the writing of the scientific article, while the review of the article was carried out by the authors Lucas Guimarães Abreu and Fernando Oliveira Costa. All authors approved the final version submitted to the Journal of Clinical and Experimental Dentistry.

\section{Conflict of Interest}

No potential conflict of interest relevant to this article was reported. 